\title{
On the Characteristic Acceleration of MOND
}

\section{Barbaro Quintero-Leyva}

Miami, FL, USA

Email: doserate2002@yahoo.com

How to cite this paper: Quintero-Leyva, B. (2017) On the Characteristic Acceleration of MOND. Open Access Library Journal, 4: e3576.

https://doi.org/10.4236/oalib.1103576

Received: April 1, 2017

Accepted: April 23, 2017

Published: April 26, 2017

Copyright (๑) 2017 by author and Open Access Library Inc.

This work is licensed under the Creative Commons Attribution International License (CC BY 4.0).

http://creativecommons.org/licenses/by/4.0/

\begin{abstract}
The impact of the geometry on the gravitational force field and the impact of the mass beyond the test particle location were assessed in the rotation curve calculation of a hypothetical thin disk galaxy using Newtonian and Mondian dynamics. An effective equation for the characteristic acceleration of MOND was obtained based on the Mestel's finite disk by normalizing the line mass density to the total mass of the galaxy and equating it to the theoretical value.
\end{abstract}

\section{Subject Areas}

Classical Mechanics, Classical Physics, Mechanics, Modern Physics, Theoretical Physics

\section{Keywords}

Dark Matter, Galaxies: Kinematics and Dynamics, Cosmology

\section{Introduction}

In 1933 ref. [1] concerning the red shift of extragalactic nebulae reported that the magnitude of the measured velocity dispersion of the Coma cluster implies much more mass than the mass estimated from the cluster luminosity-mass because the calculation of the velocity dispersion from the luminosity mass using the virial theorem yielded much lower velocity dispersion. He inferred, as one possibility, the presence of dark matter in greater amount than the luminous mass to explain the discrepancy. In 1937 ref. [2] provided more details.

In the 1970s the need for undetected additional mass in the outer part and well beyond the optical edge of the galaxy where the rotation curves showed an effective flat profile, started emerging ([3] and references therein).

To explain the dark matter problem, many scientists were in the search for a particle or object that does not interact electromagnetically while it does gravitationally. Two efforts in this direction are: WIMP (weakly interacting massive particle: non-baryonic; it has not been detected) and MACHO (massive astro- 
nomical compact halo objects: baryonic; according to [3], it does not account for the total needed dark matter in, for example, the Milky Way).

A modification of the Newtonian dynamic (MOND) was proposed in 1983 [4] [5] [6], as an alternative to the search for WIMP and MACHO, in such a way that for very low acceleration regime an asymptotically flat rotation curve is obtained well outside the optical edge of spiral galaxies and for very high acceleration the Newtonian dynamics is recovered. MOND assumes the existence of a characteristic acceleration about $10^{-10} \mathrm{~ms}^{-2}$ which is about $1 / 6$ of the current acceleration of the universe. MOND predicted a Tully-Fisher relation (TFR) of the form $M \propto V^{4}$ where $M$ and $V$ are the galaxy mass and the asymptotically flat circular velocity respectively.

In this work an equation is obtained that yields a value very close to the characteristic acceleration of MOND for disk galaxies, and the derivation is based on the use of a normalized-finite Mestel disk surface density using Newtonian dynamics.

\section{Rotation Curve Modeling}

\subsection{Homogeneous Sphere and Disk: Newtonian and Mondian Model}

MOND described as a modification of the Newton's $2^{\text {nd }}$ law can be written as [4]:

$$
F_{g}=\operatorname{ma\mu }\left(a / a_{0}\right)
$$

where

$F_{g}$ : Newtonian gravitational force acting on a test particle of mass $m$

$a$ : Newtonian acceleration of $m$

$\mu\left(a / a_{0}\right)$ : An interpolating function that is equal to $a / a_{0}$ for $a \ll a_{0}$ and is equal to 1 when $a \gg a_{0}$

$a_{0}:$ MOND characteristic acceleration.

In this work the simple interpolating function $\mu(x)=\frac{x}{1+x}$ proposed in [7] is used, it can be written as

$$
\mu\left(a, a_{0}\right)=\frac{a}{a+a_{0}}
$$

Substituting Equation (2) into Equation (1) and assuming the establishment of an acceleration given by $\frac{v^{2}}{r}$ one gets:

$$
v^{4}-F(r) r v^{2}-F(r) r^{2} a_{0}=0
$$

$v$ : Circular speed

$F(r)$ : Force per unit of test particle mass

$r$ : Distance from the center of the system to the location of the test particle.

If the impact of the geometry on the force field and the effect of the mass beyond the location of the test particle are neglected then $F(r)=\frac{G M(r)}{r^{2}}$ and Equation (3a) becomes 


$$
v^{4}-G M(r) \frac{v^{2}}{r}-G M(r) a_{0}=0
$$

$G$ : Newton gravitational constant.

Figure 1 shows the results of the calculation using Equation (3b) for a hypothetical disk galaxy (Disk-MOND in light blue) with the following data: disk radius: $R_{g}=16 \mathrm{kpc}$, constant disk thickness: $h=0.0016 \mathrm{kpc}$ (to stress the impact of the disk), mass: $M=1.0 \times 10^{11}$ solar masses $\left(M_{\text {sun }}=1.989 \times 10^{30} \mathrm{~kg}\right)$, characteristic acceleration $\left(a_{0}\right): 1.16 \times 10^{-10} \mathrm{~m} \cdot \mathrm{s}^{-2}$. The figure also shows the results for a homogeneous sphere (in red, it was modeled as a disk of variable thickness to be consistent with next calculations), for the constant thickness-disk (in green), for Keplerian dynamics (in dark blue), for Sphere-Mondian dynamics (magenta), and for Point-Mondian dynamics (in brown) where $M(r)=M$ was used in Equation (3b) i.e.

$$
v^{4}-G M \frac{v^{2}}{r}-G M a_{0}=0
$$

$M$ : Total mass of the galaxy assumed to be concentrated at its center.

Note from Figure 1 that at the edge of the galaxy, the disk yields the same speed as the sphere does because the impact of the geometry on the force field is neglected (later this impact will be considered). It can be seen that MOND in addition to yields an asymptotic flat rotational curve with circular speed significantly larger than the Keplerian model, it also raises the velocity profile in the interior of the galaxy which is consistent with the alternative need for dark matter inside the galaxy to explain experimental results (see [7] [8] for the case of the Milky Way). Note also that as should be expected, the Newton theorem regarding the validity of modeling a homogeneous sphere as a point mass for locations outside the sphere is verified even for the Sphere-MOND model.

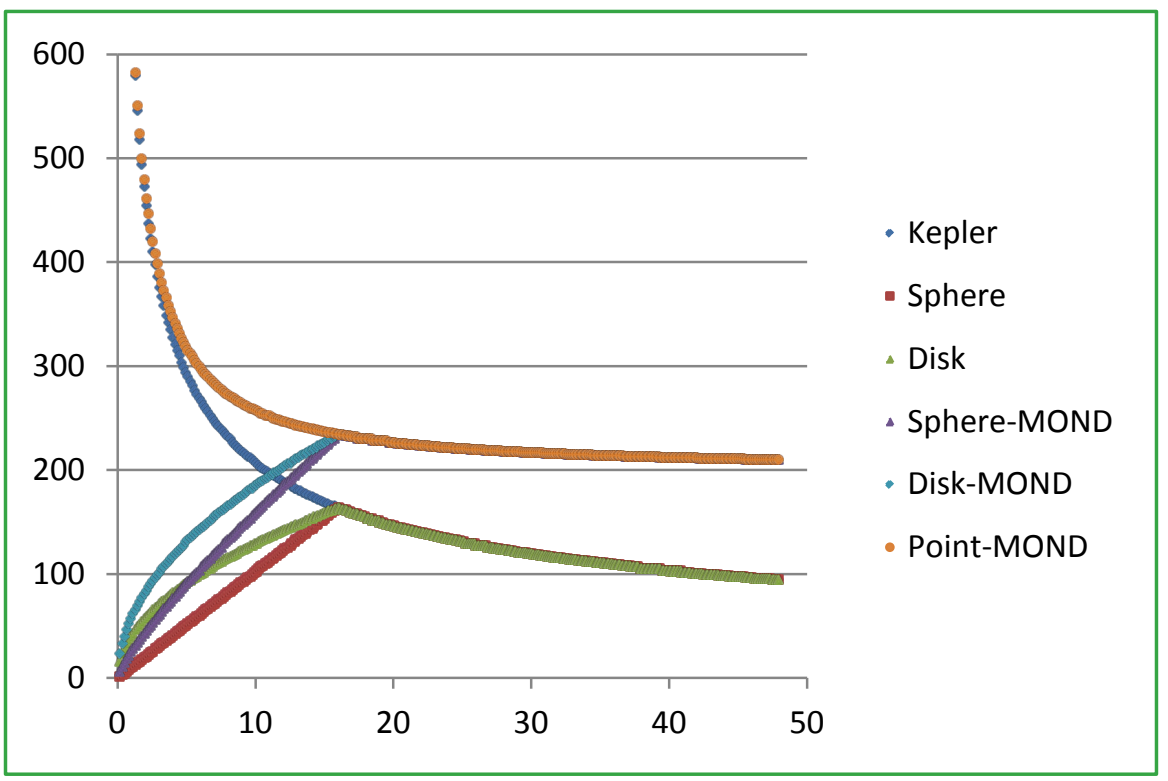

Figure 1. $V(\mathrm{~km} / \mathrm{s})$ vs. $r(\mathrm{kpc})$ per Equation $(3 \mathrm{~b})$. Neither the geometry impact on the force field nor the mass beyond the test particle location is considered. 
Figure 2 shows the results for the same cases of Figure 1 but accounting for both the impact of the geometry on the force field and the effect of the mass beyond the location of the test particle. The force per unit mass for an idealized disk galaxy was modeled following [9] as

$$
F(r) \approx G f(r), \quad f(r)=\sum_{i=1}^{N_{r}} \sum_{j=1}^{N_{a}} \frac{\Delta m_{i, j}}{\sqrt{r_{c i, j}^{2}+\left(h_{i} / 2\right)^{2}}} \frac{r-r_{s i} \cos \left(\theta_{j}\right)}{r_{c i, j}^{2}}
$$

where

$N_{r}$ : Number of radial partitions (rings)

$N_{a}$ : Number of polar partitions (sectors)

$\Delta m_{i, j}$ : Mass inside the volume $i, j: \Delta m_{i, j} \approx \rho_{i, j} h_{i} r_{i} \Delta \theta_{j} \Delta r_{i}$ with $r_{i}=r_{i-1}+\Delta r_{i}$

$r_{c i, j}$ : Distance from the center of the ring $i$ to the test particle:

$r_{c i, j}=\sqrt{\left(r_{s i} \sin \left(\theta_{j}\right)\right)^{2}+\left(r-r_{s i} \cos \left(\theta_{j}\right)\right)^{2}}$

$r_{s i}$ : Distance from the center of the galaxy to the center of the shell $i$ :

$r_{s i}=r_{i}-\Delta r_{i} / 2$

$h_{i, j}$ : Average thickness (height) of the disk at ring $i$ and sector $j$.

$\theta_{j}$ : Azimuthal angle at sector $j$ : For uniform partition $\theta_{j}=(j-1 / 2) \Delta \theta$ :

$\Delta \theta=\frac{2 \pi}{N_{a}}$

$f(r)$ was calculated using 100 rings and 360 sectors.

Note from Figure 2 that Equation (3b) (Point-MOND model) is only valid for the region beginning about where the Disk model is becoming Keplerian and that it describes a Keplerian regime only up to about $4 \mathrm{kpc}$ where the acceleration is much greater than $a_{0}$. Inspection of the acceleration results for the disk-MOND model indicates that $a_{0}$ happens in two locations, one about 12 $\mathrm{kpc}$ (inside the galaxy) and the other about $19 \mathrm{kpc}$ (outside the galaxy).

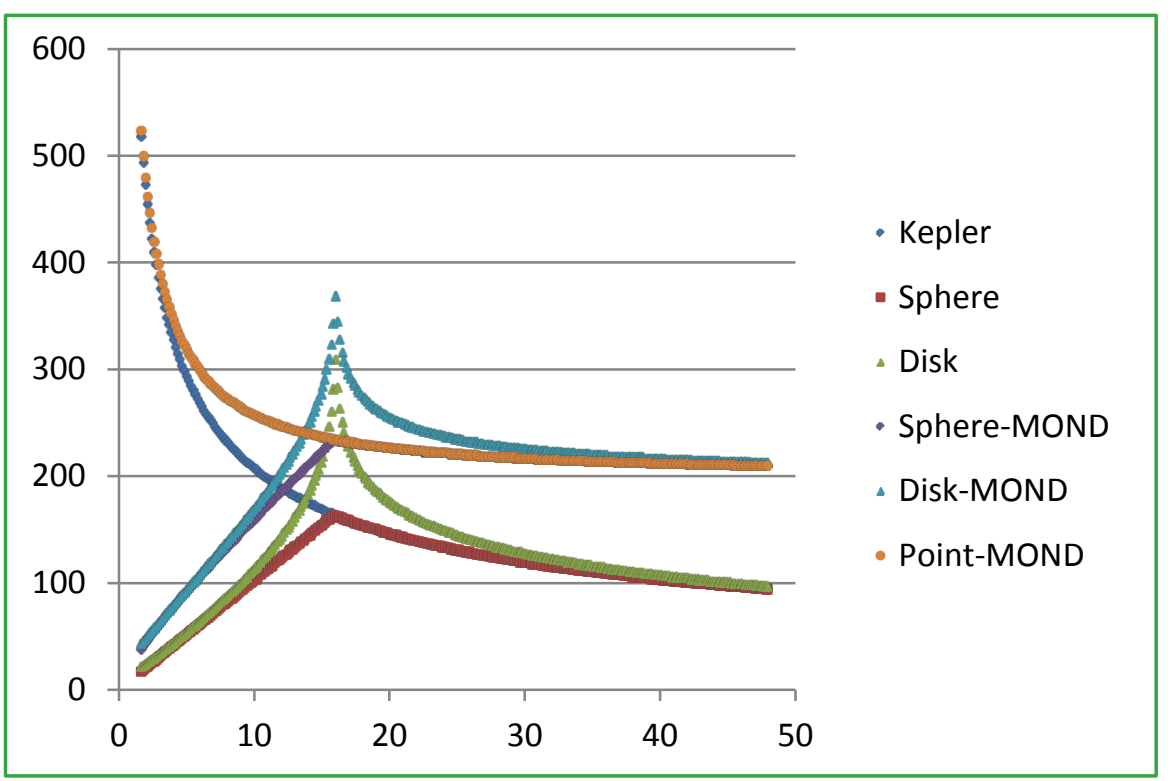

Figure 2. $v(\mathrm{~km} / \mathrm{s})$ vs. $r(\mathrm{kpc})$. The geometry impact on the force field and the mass beyond the test particle location are considered. 
Note also from Figure 2 that around the edge of the galaxy, unlike in Figure 1 , the disk (even without the MOND effect) yields significantly higher speed than what the sphere does, this is consistent with similar remarks previously made e.g. [8] and [10] where a geometrical impact on the field force was assessed.

To illustrate only the impact of the geometry on the force field, the mass beyond the location of the test particle was ignore in $f(r)$ and the results are shown in Figure 3. It can be seen that the impact is to raise significantly the speed of the disk model (with respect to the sphere model) monotonously from the interior toward the edge of the galaxy and to break the linear behavior of the speed profile of the sphere. The linear behavior is restored if the mass beyond the test particle is considered (Figure 2). Note also that the shapes of the Disk models are very different with respect the results shown in Figure 2 (concave vs. convex).

The fact that Equation (3c) yields the same asymptotic circular speed that the Disk-MOND model does, implies that the value of the asymptotic speed is independent of the details of the geometry and mass distribution inside the galaxy in question as is confirmed by the approach of the Sphere-MOND model to the same asymptotic circular speed of the Disk-MOND model. However the maximum speed and its location depend on the galaxy mass distribution as can be seen from comparing Figure 2 and Figure 4 (next section).

\subsection{Freeman and Mestel Disk}

For the exponential models of Figure 4, it was used a surface mass density of the form:

$$
\rho_{s}(r)=\rho_{s 0} \mathrm{e}^{-\alpha r} \text { for } r \leq R_{g}, 0 \text { otherwise. }
$$

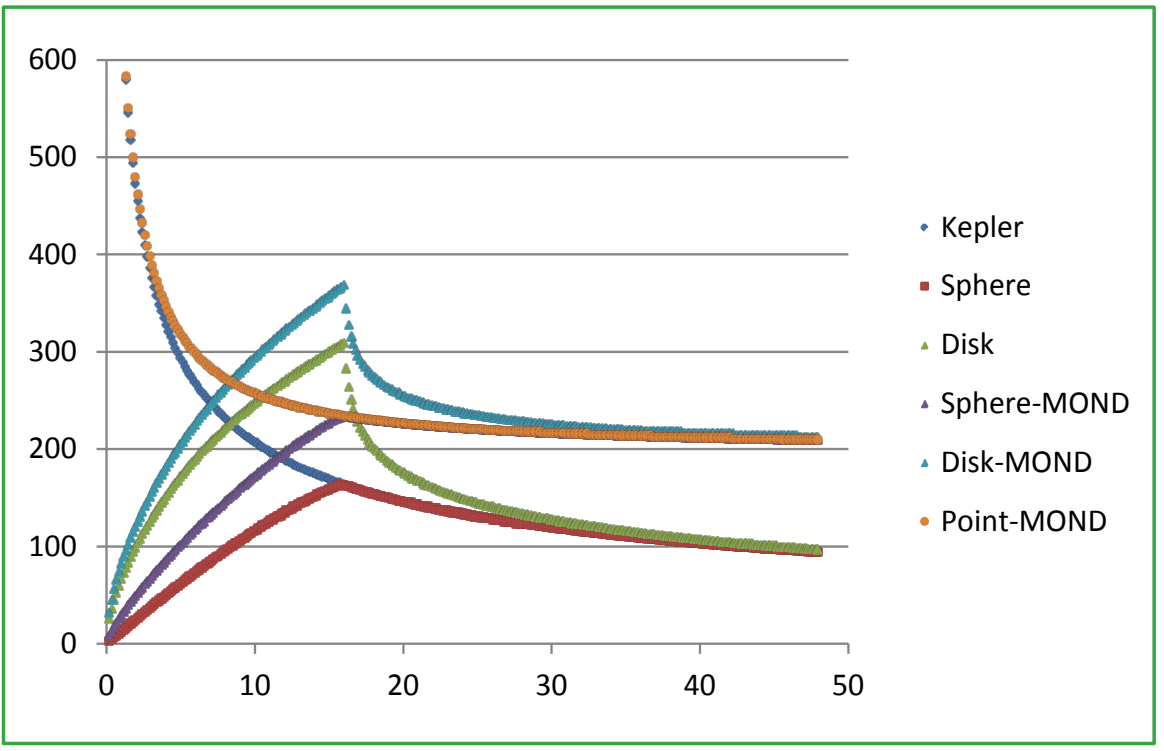

Figure 3. $v(\mathrm{~km} / \mathrm{s})$ vs. $r(\mathrm{kpc})$. The impact of the geometry on the force field is considered. The mass beyond the location of the test particle is neglected. 


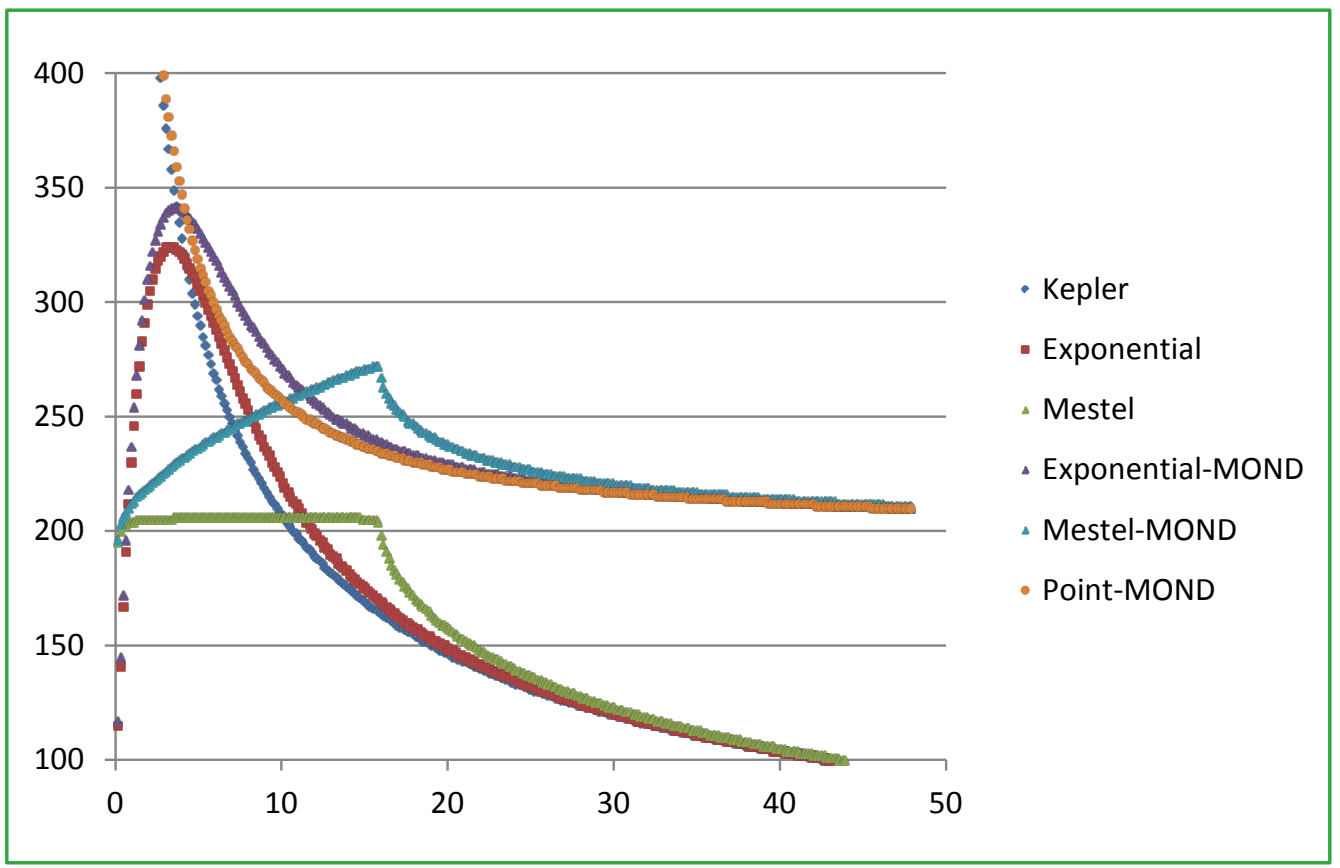

Figure 4. $v(\mathrm{~km} / \mathrm{s})$ vs. $r(\mathrm{kpc})$. The geometry impact on the force field and the mass beyond the test particle location are considered.

Here $\rho_{s 0}=\frac{M}{2 \pi \sum_{i=1}^{N_{r}} \mathrm{e}^{-\alpha r_{i}} r_{i} \Delta r_{i}}, \quad \alpha^{-1}=\frac{R_{g}}{10} \Rightarrow \Delta m_{i, j} \approx \rho_{s 0} \mathrm{e}^{-\alpha r_{i}} r_{i} \Delta \theta_{j} \Delta r_{i}$. This is a

Freeman type exponential surface density [11], which is however truncated at the edge of the galaxy and normalized per $\rho_{s 0}$.

It is noted in that figure that the exponential models do not show discontinuity, however it could appear for other values of $\alpha$. Note also that at about the edge of the galaxy, unlike in Figure 2, the exponential-Mondian model is close to the point-Mondian model and that the exponential disk-model is close to the Keplerian model. These observations suggest that the Newton theorem related with the equivalence of modeling a homogeneous sphere as a point mass, perhaps could be extended to some non-spherical systems like this one by fine tuning $\alpha$.

Figure 4 also shows the results using the Mestel finite disk-surface density given by [12]: $\rho_{s}(r)=\frac{\rho_{l 0}}{r}\left(1-\frac{2}{\pi} \sin ^{-1}\left(r / R_{g}\right)\right)$ for $r \leq R_{g}, 0$ otherwise. The linear mass density is normalized in this work according to $\rho_{l 0}=\frac{M}{2 \pi \sum_{i=1}^{N_{r}}\left(1-\frac{2}{\pi} \sin ^{-1}\left(r / R_{g}\right)\right) \Delta r_{i}} \Rightarrow \Delta m_{i, j} \approx \rho_{l 0}\left(1-\frac{2}{\pi} \sin ^{-1}\left(r / R_{g}\right)\right) \Delta \theta_{j} \Delta r_{i}$.

It is striking to see that the plateau of that figure approximately points to the asymptotic circular speed of MOND (this does not happen for a finite disk when $\rho_{s}(r)=\frac{\rho_{l 0}}{r}$ is used in $\left.f(r)\right)$. This strongly suggests that the BTF relation for thin disk galaxies could be quantified using conventional dynamics: The line 
mass density, $\rho_{l 0}$, using integrals instead of sums can be expressed as $\rho_{l 0}=\frac{M}{2 \pi R_{g}-4 \int_{0}^{R_{g}} \sin ^{-1}\left(r / R_{g}\right) \mathrm{d} r}=\frac{M}{4 R_{g}}$, Mestel's theoretical value is $\rho_{l 0}=\frac{v^{2}}{2 \pi G}$ therefore

$$
v^{4}=\left(\frac{\pi}{2} G \frac{M}{R_{g}}\right)^{2}
$$

Equation (3c) for very low acceleration becomes

$$
v^{4}=G M a_{0}
$$

For the values of $M, R_{g}$, and $a_{0}$ used the circular speed for Mestel is 205 $\mathrm{km} / \mathrm{s}$ vs. $198 \mathrm{~km} / \mathrm{s}$ for MOND. This remarkable closeness suggests the equality of Equations (4a) and (4b) taken

$$
a_{0}=\frac{\pi^{2}}{4} G \frac{M}{R_{g}^{2}}
$$

which yields $a_{0} \approx 1.3 \times 10^{-10} \mathrm{~m} / \mathrm{s}^{2}$ vs. $a_{0} \approx 1.2 \times 10^{-10}$ used for MOND. Note that the equation above is the surface gravity amplified by $\frac{\pi^{2}}{4}$. If $a_{0}=c H_{0} / k$ then $k \approx 5$ in this example. Ref. [13] determined $\frac{M}{R_{g}^{2}}$ to be approximately 0.5 $\mathrm{kg} / \mathrm{m}^{2}$ for a set of 26 disk galaxies. In this case $a_{0} \approx 8.2 \times 10^{-11} \mathrm{~m} / \mathrm{s}^{2}$ and $k \approx 8.5$. It could be worthy to accurately determine $\frac{M}{R_{g}^{2}}$ for all known isolated galaxies.

Note that from Equation (4c) $R_{g}=\frac{\pi}{2} \sqrt{\frac{G M}{a_{0}}}$ which is a rough estimate of the effective radius of a near-isothermal system indicated in [14]. Note that even though Equation (4c) depends linearly on $M$ it also depends on $R_{g}^{2}$, the ratio of the two does not change significantly compared to the change of the galaxy mass alone. Note that the derivation to obtain Equation (4c) does not provide an explanation of the MOND acceleration but the numerical coincidence of that equation with the characteristic acceleration could point towards a theory containing Equation (4c).

It is notified that if the Newtonian balance equation described in [15] for a thin disk is discretized to the following dimensional form:

$f_{F G}(r)=\sum_{i=1}^{N_{r}} \sum_{j=1}^{N_{a}} \frac{r-r_{i} \cos \left(\theta_{j}\right)}{\left(r_{i}^{2}+r^{2}-2 r_{i} r \cos \left(\theta_{j}\right)\right)^{3 / 2}} \Delta m_{i, j}$, all figures above are reproduced fairly well.

\subsection{Additional Remarks}

Considering all figures it is realized the need for accurate modeling to predict and/or fit experimental data. Neglecting the geometry impact on the force field and/or the mass beyond the location of the test particle could confirm or falsify a 
theory that modifies (or not) the current verified gravitational/inertial laws or alternative it could under (over) estimate the amount of dark matter needed to explain the experimental results.

Even though the rotation curve in the interior of disk galaxies have been previously modeled with Newtonian dynamics e.g. [13] [15] [16] [17], the large circular velocity at locations well beyond the optical length of the galaxy as indicated in ([3] and references therein, [7] [8] [13] [16]), cannot be explained with Newtonian dynamics unless the existence of dark matter is assumed and/or other non-gravitational processes are present. Because none of these possibilities have been directly verified, the alternative option of a modification of the Newton law of gravitation and/or Newton $2^{\text {nd }}$ law should not be ruled out. It is however very curious that Equation (4c) yields acceleration values remarkably close to MOND's $a_{0}$ using Newtonian dynamics.

It is puzzling that in [17] the mass distribution of the NGC 4736 galaxy was assessed using a thin disk model (covering remote parts of the galaxy) without the need for dark matter or modified dynamics but note from figures above that about twice the radius of the galaxy the velocity is still declining.

\section{Summary}

The impact of the geometry on the gravitational force field and the impact of the mass beyond the test particle location were assessed in the rotation curve calculation of a hypothetical thin disk galaxy using Newtonian and Mondian dynamics.

Based on the results of the normalized-truncated exponential surface mass density model it is suggested that the Newton theorem related with the equivalence of modeling a homogeneous sphere as a point mass just after the edge of the galaxy, perhaps could be extended to some non-spherical systems by fine tuning $\alpha$.

An effective equation for the characteristic acceleration of MOND for disk galaxies was derived from the Mestel's finite razor thin disk model by normalizing the line mass density to the mass of the galaxy and equating it to the theoretical value.

It was illustrated the need for an accurate modeling to predict and/or fit experimental data.

\section{References}

[1] Zwicky, F. (1933) The Red Shift of Extragalactic Nebulae. Republication, Springer Science + Business Media, Berlin.

[2] Zwicky, F. (1937) On the Masses of Nebulae and Clusters of Nebulae. Astrophysical Journal, 86, 217. https://doi.org/10.1086/143864

[3] Bertone, G. and Hooper, D. (2016) A History of Dark Matter. FERMILABPUB-16-157-A.

[4] Milgrom, M. (1983) A Modification of the Newtonian Dynamics as a Possible Alternative to the Hidden Mass Hypothesis. Astrophysical Journal, 270, 365-370. https://doi.org/10.1086/161130

[5] Milgrom, M. (1983) A Modification of the Newtonian Dynamics-Implications for 
Galaxies. Astrophysical Journal, 270, 371-383. https://doi.org/10.1086/161131

[6] Milgrom M. (1983) A Modification of the Newtonian Dynamics-Implications for Galaxy Systems. Astrophysical Journal, 270, 384-389.

https://doi.org/10.1086/161132

[7] Famey, B. and Binney, J. (2005) Modified Newtonian Dynamics in the Milky Way. Monthly Notices of the Royal Astronomical Society, 363, 603-608. https://doi.org/10.1111/j.1365-2966.2005.09474.x

[8] Binney, J. and Tremaine, S. (2008) Galactic Dynamics. 2nd Edition, Princeton University Press, Princeton.

[9] Nicholson, K.F. (2007) Galactic Mass Distribution without Dark Matter or Modified Newtonian Mechanics.

[10] Krizek, M., Krizek, F. and Somer, L. (2016) Dark Matter and Rotation Curves of Spiral Galaxies. Bulgarian Astronomical Journal, 25, 64.

[11] Freeman, K.C. (1970) On the Disks of Spiral and S0 Galaxies. The Astrophysical Journal, 160, 811. https://doi.org/10.1086/150474

[12] Mestel, L. (1963) MINRAS, 126.553M. On the Galactic Law of Rotation. Provided by the NASA Astrophysics Data System.

[13] Schultz, E. (2017) Scaling Relations of Mass, Velocity and Radius for Disk Galaxies.

[14] Sanders, R.H. and McGaugh, S.S. (2002) Modified Newtonian Dynamics as an Alternative to Dark Matter. Annual Review of Astronomy and Astrophysics, 40, 263 317.

[15] Feng, J.Q. and Gallo, C.F. (2008) Galactic Rotation Described with Various ThinDisk Gravitational Model.

[16] Schultz, E. (2012) The Gravitational Force and Potential of the Finite Mestel Disk. The Astrophysical Journal, 747, 106. https://doi.org/10.1088/0004-637X/747/2/106

[17] Jalocha, J., Bratek, L. and Kutschera, M. (2008) Is Dark Matter Present in NGC 4736? An Iterative Spectral Method for Finding Mass Distribution in Spiral Galaxies. The Astrophysical Journal, 679, 373-378. https://doi.org/10.1086/533511

\section{Open Access Library}

Submit or recommend next manuscript to OALib Journal and we will provide best service for you:

- Publication frequency: Monthly

- 9 subject areas of science, technology and medicine

- Fair and rigorous peer-review system

- Fast publication process

- Article promotion in various social networking sites (LinkedIn, Facebook, Twitter, etc.)

- Maximum dissemination of your research work

Submit Your Paper Online: Click Here to Submit

Or Contact service@oalib.com 